\title{
ON TWO LEMMAS OF BROWN AND SHEPP HAVING APPLICATION TO SUM SETS AND FRACTALS, II
}

\author{
C. E. M. PEARCE ${ }^{1}$ and J. PEČARIĆ ${ }^{1,2}$
}

(Received 30 June 1994)

\begin{abstract}
Simple proofs are given of improved results of Brown and Shepp which are useful in calculations with fractal sets. A new inequality for convex functions is also obtained.
\end{abstract}

\section{Introduction}

Recently there has been a resurgence of interest in sum sets, which have, inter alia, application to fractals, iterated function systems and dynamical systems (see the authors [2] for some select references in the area). The calculation of associated Hausdorff dimensions and Hausdorff measures and other properties can be delicate. In [1], Brown and Shepp provided two key lemmas which have proved valuable in making available a number of simple calculations in this area. Improvements of the results of Brown and Shepp were obtained in [2]. Further generalizations of these results are given in [3].

In particular, let $E_{i}$ be a non-empty set and $L_{i}$ a class of nonnegative functions $f_{i}: E_{i} \rightarrow \mathbf{R}(i=1,2)$. We consider functionals $A_{i}: L_{i} \rightarrow \mathbf{R}$ which satisfy the following conditions for $i=1,2$.

(a) $f_{i} \in L_{i} \Longrightarrow A_{i}\left(f_{i}\right) \geq 0$.

(b) $f_{i} \in L_{i}, \lambda_{i}>0 \Longrightarrow \lambda_{i} f_{i} \in L_{i}$ and $A_{i}\left(\lambda_{i} f_{i}\right)=\lambda_{i} A_{i}\left(f_{i}\right)$.

(c) $1 \in L_{i}$, that is, if $f_{i}(t)=1 \forall t \in E_{i}$ then $f_{i} \in L_{i}$.

(d) $f_{i}, g_{i} \in L_{i}$ with $f_{i}\left(t_{i}\right) \geq g_{i}\left(t_{i}\right) \quad\left(\forall t_{i} \in E_{i}\right) \quad \Longrightarrow \quad A_{i}\left(f_{i}\right) \geq A_{i}\left(g_{i}\right)$.

(e) $\dot{A}_{i}\left(f_{i}+g_{i}\right) \leq A_{i}\left(f_{i}\right)+A_{i}\left(g_{i}\right) \quad\left(f_{i}, g_{i} \in L_{i} \Longrightarrow f_{i}+g_{i} \in L_{i}\right)$.

Then we have the following

\footnotetext{
'Dept. of Applied Maths, The University of Adelaide, South Australia 5005.

${ }^{2}$ Faculty of Textile Technology, University of Zagreb, Zagreb, Croatia.

(C) Australian Mathematical Society, 1996, Serial-fee code 0334-2700/96
} 
THEOREM A. Let $f_{i}: L_{i} \rightarrow(0, \infty) \quad(i=1,2)$ be real functions and let the functionals $A_{i}(i=1,2)$ satisfy the five conditions above. Further, let $s_{i}, t_{i}(i=0,1,2)$ be positive numbers such that $a s_{i}^{-1}+b t_{i}^{-1}=1$ for positive constants $a, b$ and $s_{i} \leq s_{0} \leq s_{2}$. Then

$$
A_{1}\left(f_{1}^{s_{0}}\right)^{1 / s_{0}} A_{2}\left(f_{2}^{t_{0}}\right)^{1 / t_{0}} \leq \max _{i=1,2}\left\{A_{1}\left(f_{1}^{s_{i}}\right)^{1 / s_{i}} A_{2}\left(f_{2}^{t_{i}}\right)^{1 / t_{i}}\right\} .
$$

In proving this theorem we used Lemma 1 below from [4] and Theorem B.

LEMMA 1. If $f_{i}^{r} \in L_{i}(i=1,2)$ for all $r \in(0, \infty)$, then the functions

$$
G_{i}(r)=A_{i}\left(f_{i}^{r}\right) \quad(i=1,2)
$$

are logarithmically convex on $(0, \infty)$, that is, the functions $\log G_{i}(r)$ are convex.

THEOREM B. Suppose that positive numbers $s_{i}, t_{i}$ satisfy $a s_{i}^{-1}+b t_{i}^{-1}=1(i=0,1,2)$ for positive constants $a_{i}, b_{i}$ and $s_{1} \leq s_{0} \leq s_{2}$. If $f, g:(0, \infty) \rightarrow \mathbf{R}$ are convex functions, then

$$
\frac{f\left(s_{0}\right)}{s_{0}}+\frac{g\left(t_{0}\right)}{t_{0}} \leq \max _{i=1,2}\left\{\frac{f\left(s_{i}\right)}{s_{i}}+\frac{g\left(t_{i}\right)}{t_{i}}\right\} .
$$

The following generalization of Theorem $B$ is also given in [3].

THEOREM C. Suppose that positive numbers $s_{i, j}(i=0,1,2 ; j=1, \ldots, n)$ satisfy $s_{1, j} \leq s_{0, j} \leq s_{2, j}(j=1, \ldots, n)$ and $a_{j} s_{i, 1}^{-1}+b_{j} s_{i, j}^{-1}=1(i=0,1,2 ; j=2, \ldots, n)$ for positive constants $a_{j}, b_{j}(j=2, \ldots, n)$. If $f_{j}:(0, \infty) \rightarrow \mathbf{R},(j=1, \ldots, n)$ are convex functions, then

$$
\sum_{j=1}^{n} f_{j}\left(s_{0, j}\right) / s_{0, j} \leq \max _{i=1,2}\left\{\sum_{j=1}^{n} f_{j}\left(s_{i, j}\right) / s_{i, j}\right\} .
$$

Here we shall give simpler proofs of Theorems B and C.

\section{Results}

Our proofs stem from the following lemma, which is of some interest in its own right. For example, it implies from Lemma 1 that the functions

$$
H_{i}(r)=A_{i}\left(f_{i}^{1 / r}\right)^{r} \quad(i=1,2)
$$

are logarithmically convex or that the means

$$
M_{i}(r)=A_{i}\left(f_{i}^{r}\right)^{1 / r} \quad(i=1,2)
$$

are logarithmically convex functions of $1 / r$. 
LEMMA 2. Suppose $f:(0, \infty) \rightarrow \mathbf{R}$. Then $f$ is a convex function if and only if the function $F$ given by

$$
F(x)=x f(1 / x)
$$

is convex.

PROOF. First suppose that $f$ is convex. Then if $x<y<z$, we have

$$
(z-x) f(y) \leq(y-x) f(z)+(z-y) f(x) .
$$

For $b>a>0$, set $z=1 / a, x=1 / b, y=1 /[\lambda a+(1-\lambda) b]$, where $\lambda \in(0,1)$. Then (2) becomes

$$
\begin{aligned}
\left(\frac{1}{a}-\frac{1}{b}\right) f\left(\frac{1}{\lambda a+(1-\lambda) b}\right) \leq & \left(\frac{1}{\lambda a+(1-\lambda) b}-\frac{1}{b}\right) f\left(\frac{1}{a}\right) \\
& +\left(\frac{1}{a}-\frac{1}{\lambda a+(1-\lambda) b}\right) f\left(\frac{1}{b}\right),
\end{aligned}
$$

that is,

$$
[\lambda a+(1-\lambda) b] f\left(\frac{1}{\lambda a+(1-\lambda) b}\right) \leq \lambda a f\left(\frac{1}{a}\right)+(1-\lambda) b f\left(\frac{1}{b}\right),
$$

or

$$
F(\lambda a+(1-\lambda) b) \leq \lambda F(a)+(1-\lambda) F(b) .
$$

Therefore $F$ also is convex.

Because $f(x)=x F(1 / x)$, the converse follows from the result just shown.

PROOF OF THEOREM B. Let $F$ and $G$ be two convex functions on $(0, \infty)$. Then $F(x)+G(y)$, with $a x+b y=1(a, b>0)$ is also a convex function of $x$. Hence if $u_{2} \leq u_{0} \leq u_{1}$ and $a u_{i}+b v_{i}=1$ for $i=0,1,2$, then

$$
F\left(u_{0}\right)+G\left(v_{0}\right) \leq \max _{i=1,2}\left\{F\left(u_{i}\right)+G\left(v_{i}\right)\right\} .
$$

For the functions $f$ and $g$ of Theorem B we have, by Lemma 2, that the functions given by $F(x)=x f(1 / x), G(x)=x g(1 / x)$ are convex. Thus (3) becomes

$$
u_{0} f\left(1 / u_{0}\right)+v_{0} g\left(1 / v_{0}\right) \leq \max _{i=1,2}\left\{u_{i} f\left(1 / u_{i}\right)+v_{i} G\left(1 / v_{i}\right)\right\},
$$

that is, (1) holds for $u_{i}=1 / s_{i}, v_{i}=1 / t_{i}(i=0,1,2)$. 
We now prove a generalization of Theorem $\mathrm{C}$.

THEOREM 1. Suppose that positive numbers $u_{i, j}(i=0,1,2 ; j=1, \ldots, n)$ satisfy

$$
u_{1, j} \geq u_{0, j} \geq u_{2, j}(1 \leq j \leq n) \quad \text { and } \quad a_{j} u_{i, 1}+b_{j} u_{i, j}=1(i=0,1,2 ; 2 \leq j \leq n)
$$

for positive constants $a_{j}, b_{j}(2 \leq j \leq n)$ : If $F_{j}:(0, \infty) \rightarrow \mathbf{R}(1 \leq j \leq n)$ are convex functions, then

$$
\sum_{j=1}^{n} F_{j}\left(u_{0, j}\right) \leq \max _{i=1,2}\left\{\sum_{j=1}^{n} F_{j}\left(u_{i, j}\right)\right\} .
$$

ProOF. From (4) we have for $u_{1, j}>u_{0, j}>u_{2, j}$ that

$$
a_{j}\left(u_{i, 1}-u_{k, 1}\right)+b_{j}\left(u_{i, j}-u_{k, j}\right)=0
$$

for each of the pairs $(i, k)=(1,0),(2,1),(2,0)$. That is, for $\lambda \in(0,1)$,

$$
\begin{aligned}
& \frac{u_{0, j}-u_{2, j}}{u_{1, j}-u_{2, j}}=\frac{u_{2,1}-u_{0,1}}{u_{2,1}-u_{1,1}} \quad(:=\lambda), \\
& \frac{u_{1, j}-u_{0, j}}{u_{1, j}-u_{2, j}}=\frac{u_{0,1}-u_{1,1}}{u_{2,1}-u_{1,1}} \quad(:=1-\lambda) .
\end{aligned}
$$

On the other hand, the functions $F_{j}$ are convex, so

$$
F\left(u_{0, j}\right) \leq \frac{u_{0, j}-u_{2, j}}{u_{1, j}-u_{2, j}} F_{j}\left(u_{1, j}\right)+\frac{u_{1, j}-u_{0, j}}{u_{1, j}-u_{2, j}} F_{j}\left(u_{2, j}\right),
$$

that is, $F\left(u_{0, j}\right) \leq \lambda F_{j}\left(u_{1, j}\right)+(1-\lambda) F_{j}\left(u_{2, j}\right)$.

Summation gives

$$
\begin{aligned}
\sum_{j=1}^{n} F_{j}\left(u_{0, j}\right) & \leq \lambda \sum_{j=1}^{n} F_{j}\left(u_{1, j}\right)+(1-\lambda) \sum_{j=1}^{n} F_{j}\left(u_{2, j}\right) \\
& \leq \max _{i=1,2}\left\{\sum_{j=1}^{n} F_{j}\left(u_{i, j}\right)\right\} .
\end{aligned}
$$

Theorem $\mathrm{C}$ follows in the particular case $F_{j}(x)=x f_{j}(1 / x)$ and $u_{i, j}=1 / s_{i, j}(i=$ $0,1,2 ; 1 \leq j \leq n)$. 
REMARK 1. Lemma 2 can be generalized as follows.

For an integer $n \geq 1$, the reciprocal transformation of order $n$ of a function $f$ whose domain is an interval of positive numbers is the function $\phi_{n}$ given by

$$
\phi_{n}(t)=(-1)^{n} t^{n-1} f(1 / t) .
$$

The reciprocal transformation of order $n$ of $\phi_{n}$ is evidently $f$. We have the following. The reciprocal transformation of order $n$ preserves $n$-convexity, that is, $\phi_{n}$ is $n$-convex if and only if $f$ is $n$-convex.

Recall that a function $f$ is $n$-convex if, for $n+1$ distinct points $x_{n}$, we have

$$
\sum_{k=0}^{n} f\left(x_{k}\right) / \prod_{\substack{j=0 \\ j \neq k}}^{n}\left(x_{k}-x_{j}\right) \geq 0
$$

(see [5, pages 14-16]).

To establish the statement enunciated, suppose $f$ is $n$-convex and set $x_{k}=1 / t_{k}$ in (5). Simple manipulations provide

$$
\left[\prod_{j=0}^{n} t_{j}\right] \sum_{k=0}^{n}(-1)^{n} t_{k}^{n-1} f\left(1 / t_{k}\right) / \prod_{\substack{j=0 \\ j \neq k}}^{n}\left(t_{k}-t_{j}\right) \geq 0,
$$

that is,

$$
\sum_{k=0}^{n} \phi_{n}\left(t_{k}\right) / \prod_{\substack{j=0 \\ j \neq k}}^{n}\left(t_{k}-t_{j}\right) \geq 0
$$

so $\phi_{n}$ is $n$-convex too.

Since $n$-convexity coincides with ordinary convexity for $n=2$, this establishes an alternate proof for Lemma 2.

\section{References}

[1] G. Brown and L. Shepp, "A convolution inequality", in Contributions to Prob. and Stat. Essays in Honor of Ingram Olkin, (Springer, New York, 1989) 51-57.

[2] C. E. M. Pearce and J. E. Pečarić, "On two lemmas of Brown and Shepp having application to sum sets and fractals", J. Austral. Math. Soc. Ser. B 36 (1994) 60-63.

[3] C. E. M. Pearce and J. E. Pečarić, "An inequality for convex functions", J. Math. Analysis and Applic. 183 (1994) 523-527.

[4] J. E. Pečarić, "Generalization of the power means and their inequalities", J. Math. Analysis and Applic. 161 (1991) 395-409.

[5] J. E. Pečarić, F. Proschan and Y. L. Tong, Convex functions, partial orderings and statistical applications (Academic Press, Boston, 1992). 